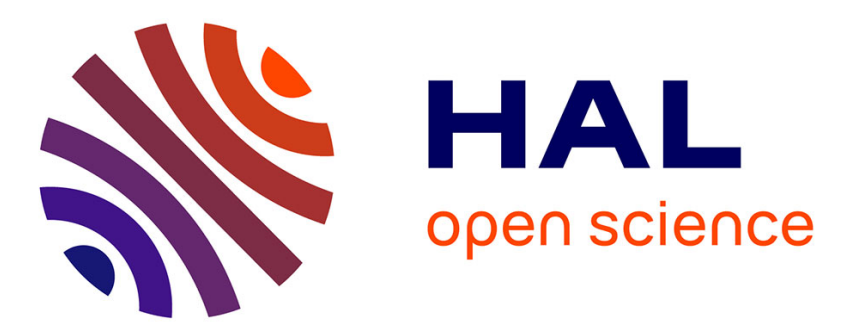

\title{
Interpolated pressure laws in two-fluid simulations and hyperbolicity
}

Philippe Helluy, Jonathan Jung

\section{To cite this version:}

Philippe Helluy, Jonathan Jung. Interpolated pressure laws in two-fluid simulations and hyperbolicity. Finite Volumes for Complex Applications VII-Methods and Theoretical Aspects, Springer, pp.37-53, 2014. hal-00957043

\section{HAL Id: hal-00957043 https://hal.science/hal-00957043}

Submitted on 7 Mar 2014

HAL is a multi-disciplinary open access archive for the deposit and dissemination of scientific research documents, whether they are published or not. The documents may come from teaching and research institutions in France or abroad, or from public or private research centers.
L'archive ouverte pluridisciplinaire HAL, est destinée au dépôt et à la diffusion de documents scientifiques de niveau recherche, publiés ou non, émanant des établissements d'enseignement et de recherche français ou étrangers, des laboratoires publics ou privés. 


\title{
Interpolated pressure laws in two-fluid simulations and hyperbolicity
}

\author{
Philippe HELLUY and Jonathan JUNG
}

\begin{abstract}
We consider a two-fluid compressible flow. Each fluid obeys a stiffened gas pressure law. The continuous model is well defined without considering mixture regions. However, for numerical applications it is often necessary to consider artificial mixtures, because the two-fluid interface is diffused by the numerical scheme. We show that classic pressure law interpolations lead to a non-convex hyperbolicity domain and failure of well-known numerical schemes. We propose a physically relevant pressure law interpolation construction and show that it leads to a necessary modification of the pure phase pressure laws. We also propose a numerical scheme that permits to approximate the stiffened gas model without artificial mixture.
\end{abstract}

\section{Introduction}

The numerical simulation of compressible two-fluid flows with Eulerian finite volume approximation has been widely studied. We refer for instance to [20, 17, 22] and included references. The Eulerian approach is very appealing compared to Lagrangian front tracking methods because it generally leads to much simpler algorithms. However, one has to circumvent the pressure oscillations that appear at the two-fluid interface with standard conservative schemes. The lack of accuracy of the Godunov scheme at contact waves is a well-known issue. It is not only observed in two-fluid simulations but also in one-fluid simulations when the single fluid satisfies a complex pressure law [8].

Another less known aspect of classic two-fluid models is that their hyperbolicity domain is generally not convex. Thus in some cases, the Godunov scheme is unstable and fails after only one time iteration.

Philippe Helluy

IRMA, University of Strasbourg and Inria Tonus e-mail: helluy @ math.unistra.fr

Jonathan Jung

IRMA, University of Strasbourg e-mail: jonathan.jung@unistra.fr 
In this paper we consider the flow of a gas and a liquid modeled by two stiffened gas equations of state. The pressure law is initially only defined in the pure phases: the mass fraction of gas $\varphi$ can take only two values $\varphi=0$ or $\varphi=1$.

For numerical reasons, the pressure law is often interpolated in an artificial mixture region $0<\varphi<1$. We show that a naive numerical interpolation always leads to the non-convexity of the hyperbolicity domain and thus to the instability of the Godunov scheme.

We then propose two alternative cures to this issue:

1. The construction of a mixture pressure law based on physical and thermodynamical arguments. We are then able to recover a convex hyperbolicity domain. But we also prove that it is necessary to modify the pressure law of the liquid phase, which cannot be a simple stiffened gas anymore.

2. For keeping the simplicity of the stiffened gas model, we propose another scheme, the Random Interface Solver (RIS) [16]. This scheme does not diffuse the mass fraction profile and allows stable computations of the two-fluid model.

Finally we present some two-dimensional numerical results obtained with the RIS scheme.

\section{Two-fluid flows and Godunov scheme}

We consider a two-fluid model (air and liquid water) written as a first order system of conservation laws

$$
\partial_{t} W+\partial_{x} F(W)=0
$$

The space variable is $x$. The time variable is $t$. We use the notations $\partial_{t}=\partial / \partial t$, $\partial_{x}=\partial / \partial x$. The conservative unknowns $(x, t) \rightarrow W(x, t)$ are

$$
W=(\rho, \rho u, \rho e, \rho \varphi)^{T},
$$

with the density $\rho$, velocity $u$, total energy $e$ and mass fraction of gas $\varphi$. The total energy $e$ is related to the internal energy $\varepsilon$ by

$$
e=\varepsilon+\frac{1}{2} u^{2} .
$$

The flux of the conservative system is given by

$$
F(W)=\left(\rho u, \rho u^{2}+p,(\rho e+p) u, \rho \varphi u\right)^{T} .
$$

The pressure law is of the form

$$
p=P(\rho, \varepsilon, \varphi) .
$$

If at the initial time $t=0$ the mass fraction $\varphi$ takes only two values 0 (pure liquid phase) and 1 (pure gas phase) then it is also true at any later time. This property 
implies that theoretically it is only necessary to provide the pressure laws $P(\rho, \varepsilon, 0)$ for the liquid and $P(\rho, \varepsilon, 1)$ for the gas. A classic choice is the stiffened gas pressure law

$$
\begin{gathered}
P(\rho, \varepsilon, \varphi)=(\gamma(\varphi)-1) \rho \varepsilon-\gamma(\varphi) \pi(\varphi), \\
\gamma(1)=\gamma_{1}>1, \quad \pi(1)=\pi_{1}=0, \quad \gamma(0)=\gamma_{2}>1, \quad \pi(0)=\pi_{2}>0 .
\end{gathered}
$$

The constants $\gamma_{1}, \gamma_{2}$ and $\pi_{2}$ are obtained from physical measurements. For instance, for air and water, we can take [1]

$$
\gamma_{1}=1.4, \quad \gamma_{2}=3, \quad \pi_{2}=8533 \times 10^{5} \mathrm{~Pa} .
$$

In the following, we consider the properties of system (1)-(6). In short, we call it the two-fluid model.

In practice, it is difficult to impose $\varphi=0$ or $\varphi=1$ in the numerical approximation. A widely used possibility (see for instance $[2,11,17,21,20,22]$ ) is to interpolate the pressure laws parameter $\gamma(\varphi)$ and $\pi(\varphi)$ for $\varphi \in] 0,1[$.

For the stiffened gas pressure law, the sound speed is given by

$$
c=\sqrt{\frac{\gamma(\varphi)(p+\pi(\varphi))}{\rho}},
$$

we thus obtain that the system (1)-(6) is hyperbolic if $W$ is in the hyperbolicity domain

$$
\Omega=\{(\rho, \rho u, \rho e, \rho \varphi), \rho \geq 0, \varphi \in[0,1], p+\pi(\varphi) \geq 0 .\}
$$

The Riemann problem for the two-fluid system consists in solving the following initial value problem

$$
\begin{gathered}
\partial_{t} W+\partial_{x} F(W)=0, \\
W(x, 0)=\left\{\begin{array}{c}
W_{L} \text { if } x<0, \\
W_{R} \text { otherwise }
\end{array}\right.
\end{gathered}
$$

The left and right constant states $W_{L}, W_{R}$ are taken into the hyperbolicity domain $\Omega$. The solution is self-similar, denoted by

$$
R\left(W_{L}, W_{R}, \frac{x}{t}\right)=W(x, t) .
$$

It is made of shock waves, rarefaction waves and contact waves, separated by constant states. It is well known that the solution of the Riemann problem is generally not unique. In order to reduce the set of solutions, we can for instance consider only shock waves that satisfy the Lax characteristic criterion. Below, we will also discuss the Lax entropy criterion.

An essential feature of the two-fluid system is that the Riemann problem admits a unique global solution that satisfies the Lax characteristic criterion whenever the left and right initial states are in the hyperbolicity domain $\Omega$. This global solution is constructed from standard wave parameterization [18]. It is sometimes necessary 
to introduce vacuum state in the gas phase (see [2] for details). The solution is not only theoretical. It can also be computed almost analytically in an efficient way.

The Riemann problem being uniquely solvable, it is then tempting to apply the Godunov scheme to the two-fluid model with arbitrary initial data.

We consider a sequence of time $t_{n}, n \in \mathbb{N}$ such that the time step $\tau_{n}=t_{n+1}-t_{n}>0$. We consider also a space step $h$. We define the cell centers by $x_{i}=i h$. The cell $C_{i}$ is the interval $] x_{i-1 / 2}, x_{i+1 / 2}[$. We consider an approximation

$$
W_{i}^{n} \simeq \frac{1}{h} \int_{x \in C_{i}} W\left(x, t_{n}\right) d x .
$$

A time step of the Godunov scheme is made of two stages:

- Step1: Exact resolution starting from approximated cell averages

$$
\begin{gathered}
\partial_{t} V+\partial_{x} F(V)=0, \\
V(x, 0)=W_{i}^{n}, \quad x \in C_{i} .
\end{gathered}
$$

- Step 2: Averaging of the exact solution

$$
W_{i}^{n+1}=\frac{1}{h} \int_{C_{i}} V\left(x, \tau_{n}\right) d x .
$$

The time marching scheme also admits a finite volume formulation

$$
W_{i}^{n+1}=W_{i}^{n}-\frac{h}{\tau_{n}}\left(F_{i+1 / 2}^{n}-F_{i-1 / 2}^{n}\right) .
$$

The numerical fluxes are computed from exact solutions of the Riemann problem

$$
F_{i+1 / 2}^{n}=F\left(R\left(W_{i}^{n}, W_{i+1}^{n}, 0\right)\right) .
$$

The time step satisfies a CFL condition

$$
\tau_{n} \leq \frac{h}{2 \lambda_{n}^{\max }},
$$

where $\lambda_{n}^{\max }$ is an upper bound of all the wave speeds in the solutions of the interface Riemann problems at time $t_{n}$.

In our application, Step 1 of the Godunov scheme is not a problem if all the $W_{i}^{n}$ are in $\Omega$ because the Riemann problem admits a unique physically relevant solution $W(x, t) \in \Omega$. Surprisingly, Step 2 is much more problematic when $\pi_{1} \neq \pi_{2}$, because of the following result [16]:

Theorem 1. Consider the two-fluid system (1)-(6) and suppose a continuous interpolation of the pressure law parameters $\varphi \rightarrow \gamma(\varphi), \varphi \rightarrow \pi(\varphi)$ for $\varphi \in[0,1]$ satisfying (7). Then, the hyperbolicity set $\Omega$ is never convex. 
The non-convexity of $\Omega$ is a big issue, because even if we have $V\left(x, \tau_{n}\right) \in \Omega$ in the averaging formula (8), we cannot conclude that $W_{i}^{n+1} \in \Omega$. In practice it is possible to construct initial data for which the Godunov scheme fails after only one iteration $[21,16]$. In conclusion, the Godunov scheme applied to the two-fluid model with $\pi_{1} \neq \pi_{2}$ is generally unstable.

Remark 1. The numerical resolution of the two-fluid model with stiffened gas pressure law has been extensively studied. When the two fluids are perfect gases (when $\left.\pi_{1}=\pi_{2}=0\right)$ the hyperbolicity set $\Omega$ is convex. In this case, the Godunov scheme is stable. But it often gives very inaccurate results in contact waves. In the literature, this behavior is called the pressure oscillation phenomenon. Let us emphasize that the instability of the Godunov scheme for $\pi_{2} \neq \pi_{1}$ and the pressure oscillations in contact waves are two different and independent shortcomings of the Godunov scheme applied to two-fluid flows.

Remark 2. A very popular method for avoiding pressure oscillations has been proposed by Abgrall and Saurel in [22]. The method relies on a non-conservative numerical resolution of the transport equation

$$
\partial_{t} \varphi+u \partial_{x} \varphi=0
$$

and a special interpolation of the pressure law coefficients $\gamma(\varphi), \pi(\varphi)$ that ensures that the pressure and the velocity remain numerically constant in contact waves. Even if this trick improves the Godunov scheme accuracy, it does not improve the stability. Indeed, it is possible to show that the hyperbolicity set, expressed in the non conservative variables

$$
\Omega^{\prime}=\{(\rho, \rho u, \rho e, \varphi), \rho \geq 0, \varphi \in[0,1], p+\pi(\varphi) \geq 0\}
$$

is also generally not convex. We can also exhibit physical initial state that leads to the failure of the Abgrall-Saurel scheme after only one iteration [16].

Remark 3. A Lax entropy $W \rightarrow U(W) \in \mathbb{R} \cup\{+\infty\}$ is a strictly convex function of $W$ associated to an entropy flux $W \rightarrow G(W)$ such that the smooth solutions of the two-fluid model satisfy the additional conservation law

$$
\partial_{t} U(W)+\partial_{x} G(W)=0 .
$$

If a system of conservation laws admits a Lax entropy, then from Mock's theorem [19], we know that the system is hyperbolic on the domain of $U$ and thus

$$
\Omega=\operatorname{Dom} U=\left\{W \in \mathbb{R}^{4}, U(W)<+\infty\right\} .
$$

Because the domain of a convex function is a convex set, we deduce that the twofluid model cannot possess a global Lax entropy.

In conclusion of this section, we have two alternatives for approximating the two-fluid model in a robust and precise way: 
1. We can abandon the stiffened gas pressure law (6) and construct another pressure law that ensures the convexity of the hyperbolicity set.

2. If we keep the stiffened gas pressure law we have to construct a scheme that is stable with respect to non-convex hyperbolicity set.

In Section 2, we investigate the first possibility, while in Section 3 we consider the second.

\section{Convex mixture}

In this section, we consider a mixture of a perfect gas and a liquid satisfying the stiffened gas pressure law. From physical entropy arguments, we construct a mixture pressure law. This pressure law is naturally associated to a convex Lax entropy of the two-fluid system. Mock's theorem then ensures the convexity of the hyperbolicity set. The construction is split into several steps.

\subsection{Construction of an extensive mixture entropy}

The pressure law is constructed as follows. First, we consider two fluids indexed by $i=1$ and $i=2$ of mass $M_{i}$, energy $E_{i}$, occupying a volume $V_{i}$. We introduce the specific heat $\chi_{i}$ of fluid $i$. Then, for $M_{i}>0, V_{i}>0$ and $E_{i}>\pi_{i} V_{i}$, the entropy function of fluid $i$ is

$$
S_{i}\left(V_{i}, E_{i}, M_{i}\right)=-\chi_{i} \gamma_{i} M_{i} \ln M_{i}+\chi_{i}\left(\gamma_{i}-1\right) M_{i} \ln V_{i}+\chi_{i} M_{i} \ln \left(E_{i}-\pi_{i} V_{i}\right) .
$$

For completely rigorous proofs, we have to define the entropies for all $\left(V_{i}, E_{i}, M_{i}\right) \in$ $\mathbb{R}^{3}$. We thus also set

$$
S_{i}\left(V_{i}, E_{i}, 0\right)=0, \quad V_{i} \geq 0, \quad E_{i} \geq \pi_{i} V_{i},
$$

and

$$
S_{i}\left(V_{i}, E_{i}, 0\right)=-\infty
$$

in all the other cases. With this definition, $S_{i}$ are concave upper semicontinous (in short: usc) functions and

$$
\begin{aligned}
\operatorname{Dom} S_{i}= & \left\{\left(V_{i}, E_{i}, M_{i}\right), V_{i}>0, E_{i}>\pi_{i} V_{i}, M_{i}>0\right\} \\
& \cup\left\{\left(V_{i}, E_{i}, 0\right), V_{i} \geq 0, E_{i} \geq \pi_{i} V_{i}\right\} .
\end{aligned}
$$

Dom $S_{i}$ are convex cones. In addition, the entropies $S_{i}$ are Positively Homegeneous function of degree 1 (in short PH1)

$$
\forall \lambda \geq 0, \forall W \in \mathbb{R}^{3}, S(\lambda W)=\lambda S(W) .
$$


We then define the entropy of the immiscible mixture by

$$
S\left(V, E, M, M_{1}\right)=\sup _{V_{1}, E_{1}}\left(S_{1}\left(V_{1}, E_{1}, M_{1}\right)+S_{2}\left(V-V_{1}, E-E_{1}, M-M_{1}\right)\right) .
$$

This formula is physically justified by the fact that the entropy is an additive quantity and by the second principle of thermodynamics: the mixture of the two fluids evolves until it reaches a maximum of entropy. For more details, we refer to $[1,15,14,16]$ and included references. Let us also observe that we do not optimize the mixture entropy with respect to $M_{1}$ because we do not consider phase transition between the liquid and the gas.

From standard convex analysis, it is possible to prove the following result [16]:

Theorem 2. Let $S$ be defined by (13), where $S_{1}$ and $S_{2}$ satisfy (9)-(11). Then $S$ is a PHI concave and usc function. Its domain is a convex cone given by

$$
\begin{aligned}
D o m S= & \left\{\left(V, E, M, M_{1}\right), V>0, E>0, M \geq M_{1} \geq 0\right\} \\
& \cup\{(V, E, 0,0), V \geq 0, E \geq 0\} .
\end{aligned}
$$

\subsection{Intensive mixture entropy and pressure law}

From the extensive PH1 entropy, we can go back to intensive variables. We have the following relations

$$
\rho=\frac{M}{V}, \tau=\frac{1}{\rho}=\frac{V}{M}, \varepsilon=\frac{E}{M}, \varphi=\frac{M_{1}}{M}, s=\frac{S}{M}, \sigma=\frac{S}{V} .
$$

We then define the intensive specific entropy

$$
s(\tau, \varepsilon, \varphi)=S(\tau, \varepsilon, 1, \varphi) .
$$

From Theorem 2, the specific entropy is a concave function. We define the pressure $p$, temperature $T$ and chemical potential $\lambda$ of the mixture by

$$
T=\frac{1}{\partial_{\varepsilon} s}, \quad p=T \partial_{\tau} s, \quad \lambda=T \partial_{\varphi} s
$$

in such a way that

$$
T d s=d \varepsilon+p d \tau+\lambda d \varphi .
$$

We can also consider the volumic entropy

$$
\sigma(\rho, \rho \varepsilon, \rho \varphi)=S(1, \rho \varepsilon, \rho, \rho \varphi)=\rho s\left(\frac{1}{\rho}, \frac{\rho \varepsilon}{\rho}, \frac{\rho \varphi}{\rho}\right) .
$$

In the same way, the volumic entropy is a concave function of $(\rho, \rho \varepsilon, \rho \varphi)$.

It is then standard $[10,12,16]$ to deduce that the quantity 


$$
U(W)=-\sigma(\rho, \rho \varepsilon, \rho \varphi), \quad \text { with } W=\left(\rho, \rho u, \rho \varepsilon+1 / 2 \rho u^{2}, \rho \varphi\right)^{T},
$$

is a convex Lax entropy associated to the entropy flux

$$
G(W)=u U(W)
$$

Our whole construction ensures that the two-fluid system with the pressure law given by (15) is necessarily hyperbolic on a convex domain and that this convex domain is simply the domain of the Lax entropy $U$.

\subsection{Explicit pressure law}

It is interesting to perform the full computations in order to see how the resulting pressure $P(\rho, \varepsilon, \varphi)$ is different from the interpolated pressure law (6). The computations are not very difficult but a little bit lengthy. They are rigorously detailed in [16].

We give the final result. Let us just mention that the same formula can be obtained by formally assuming pressure and temperature equilibrium between the two phases

$$
p_{1}=p_{1}, \quad T_{1}=T_{2} .
$$

The temperatures $T_{1}$ and $T_{2}$ of the two stiffened gases are given by the relation

$$
\chi_{i} T_{i}=1 / \partial_{\varepsilon_{i}} s_{i}\left(\tau_{i}, \varepsilon_{i}\right)=\varepsilon_{i}-\pi_{i} \tau_{i} .
$$

Of course, such equilibrium assumption has no meaning when $\varphi=0$ or $\varphi=1$ because in this case only one phase is present in the mixture. The entropy optimization procedure is more rigorous and allows handling the cases $\varphi=0$ or $\varphi=1$.

We take a density $\rho>0$, an internal energy $\varepsilon>0$. We define the heat capacity of the mixture by

$$
\chi=\chi(\varphi)=\chi_{1} \varphi+(1-\varphi) \chi_{2} .
$$

We also define the energy fraction of the mixture by

$$
\zeta=\zeta(\varphi)=\frac{\chi_{1} \varphi}{\chi}
$$

The mixture polytropic parameter is then

$$
\gamma=\gamma(\varphi)=\zeta \gamma_{1}+(1-\zeta) \gamma_{2}
$$

We also consider the following quantities

$$
\delta=-\gamma_{2} \pi_{2}, \quad r=(\delta+(\gamma-1) \rho e)^{2}-4 \delta\left(\gamma_{1}-1\right) \zeta \rho e>0 .
$$

The volume fraction of gas is then given by 


$$
\alpha=\alpha(\varphi)=\frac{\delta+(\gamma-1) \rho e-\sqrt{r}}{2 \delta} .
$$

1. If $0<\varphi<1$ then

$$
P(\rho, \varepsilon, \varphi)=(\gamma-1) \rho \varepsilon-\gamma(1-\alpha) \pi_{2}
$$

2. If $\varphi=1$ then

$$
P(\rho, \varepsilon, \varphi)=\left(\gamma_{1}-1\right) \rho \varepsilon
$$

3. If $\varphi=0$ then

$$
P(\rho, \varepsilon, 0)=\max \left(0,\left(\gamma_{2}-1\right) \rho \varepsilon-\gamma_{2} \pi_{2}\right) .
$$

The main result of this analysis is that even if the mass of gas vanishes $(\varphi=0)$ the remaining liquid does not always obey a pure stiffened gas law. When the energy is small enough, the liquid pressure vanishes. Intuitively, this means that the liquid undergoes a cavitation phenomenon. The liquid pressure cannot be negative anymore, while it was possible in the pressure law (6).

The pressure law obtained with the entropy optimization procedure ensures a convex hyperbolicity domain. It thus ensures the stability of the Godunov scheme. However, the pressure law is more complex than the stiffened gas law. For instance, in a pure liquid region, the two-fluid model can degenerate to a pressureless Euler system. This system is known to lead to theoretical and numerical difficulties. In addition, we have verified in numerical experiments that the pressure oscillation phenomenon is still present at contact waves. Therefore, we will also propose in the next section a practical numerical method for solving directly the two-fluid model with a non-convex hyperbolicity domain.

\section{A new Random Interface Solver}

In this section, we return to the simple stiffened gas pressure law (6). We define the hyperbolicity sets in the pure phases

$$
\Omega_{0}=\Omega \cap\{\varphi=0\}, \quad \Omega_{1}=\Omega \cap\{\varphi=1\} .
$$

These two sets are convex. We consider a numerical initial condition in the pure phases

$$
\forall i, W_{i}^{0} \in \Omega_{0} \cup \Omega_{1} .
$$

We show how to construct a scheme, the Random Interface Solver (RIS) that satisfies the stability condition

$$
\forall i, \forall n \geq 0, \quad W_{i}^{n} \in \Omega_{0} \cup \Omega_{1} .
$$

From the literature and the considerations above we know two things:

1. The new scheme cannot be exactly conservative at each time step; 
2. If we average the mass fraction on the cells of the initial mesh, we will introduce a numerical diffusion and certainly pressure oscillations at the interface.

In order to avoid these two pitfalls we will

1. use a random sampling strategy at the interface. It allows avoiding the diffusion of the mass fraction profile. It is not perfectly conservative, but we can prove that it is statistically conservative on long times;

2. before the random sampling, we use a Lagrangian conservative finite volume scheme at the interface. In this Lagrangian step, the mass fraction is not diffused either.

We now enter into the details of the RIS scheme. Each time step of the scheme is made of two stages: an Arbitrary Lagrangian Eulerian (ALE) step and a Projection step. The idea to combine the Glimm's scheme approach [9] and a Lagrangian scheme approach was first proposed by Chalons and Goatin in [5]. See also $[3,4,13]$.

\subsection{ALE stage}

In the first stage, we allow the cell boundary $x_{i+1 / 2}$ to move at velocity $\xi_{i+1 / 2}^{n}$. At the end of the first stage, the cell boundary is

$$
x_{i+1 / 2}^{n+1,-}=x_{i+1 / 2}+\tau_{n} \xi_{i+1 / 2}^{n} .
$$

Integrating the conservation law (1) on the moving cells, we obtain the following finite volume approximation

$$
h_{i}^{n+1,-} W_{i}^{n+1,-}-h W_{i}^{n}+\tau_{n}\left(F_{i+1 / 2}^{n}-F_{i-1 / 2}^{n}\right)=0 .
$$

The new size of cell $i$ is given by

$$
h_{i}^{n+1,-}=x_{i+1 / 2}^{n+1,-}-x_{i-1 / 2}^{n+1,-}=h+\tau_{n}\left(\xi_{i+1 / 2}^{n}-\xi_{i-1 / 2}^{n}\right) .
$$

The numerical flux is now of the ALE form

$$
F_{i+1 / 2}^{n}=F\left(W_{i+1 / 2}^{n}\right)-\xi_{i+1 / 2}^{n} W_{i+1 / 2}^{n} .
$$

The intermediate state $W_{i+1 / 2}^{n}$ is obtained by the resolution of a Riemann problem

$$
W_{i+1 / 2}^{n}=R\left(W_{i}^{n}, W_{i+1}^{n}, \xi_{i+1 / 2}^{n}\right) .
$$

In practice, $R$ can also be an approximate Riemann solver [16].

Finally, the interface velocity is defined by

$$
\xi_{i+1 / 2}^{n}=\left\{\begin{array}{cc}
u_{i+1 / 2}^{n} & \text { if }\left(\varphi_{i}^{n}-1 / 2\right)\left(\varphi_{i+1}^{n}-1 / 2\right)<0 \\
0 & \text { else. }
\end{array}\right.
$$


The numerical flux is thus a classic Godunov flux in the pure fluid. It is a Lagrangian numerical flux at the two-fluid interface.

\subsection{Projection step}

The second stage of the time step is needed for returning to the initial mesh. We have to compute on the cells $C_{i}$ of the initial mesh the averages of $W_{i}^{n+1,-}$, defined on the moved cells $\left.C_{i}^{n+1,-}=\right] x_{i-1 / 2}^{n+1,-}, x_{i+1 / 2}^{n+1,-}$ [. Instead of a standard integral averaging method, we rather consider a random sampling averaging process. We consider a pseudo random sequence $\omega_{n} \in[0,1[$ and we perform the following sampling

$$
W_{i}^{n+1}=\left\{\begin{array}{l}
W_{i-1}^{n+1,-}, \text { if } \omega_{n}<\frac{\xi_{i-1 / 2}^{n} \tau_{n}}{h}, \\
W_{i}^{n+1,-}, \text { if } \frac{\xi_{i-1 / 2}^{n} \tau_{n}}{h} \leq \omega_{n} \leq 1+\frac{\xi_{i+1 / 2}^{n} \tau_{n}}{h}, \\
W_{i+1}^{n+1,-}, \text { if } \omega_{n}>1+\frac{\xi_{i+1 / 2}^{n} \tau_{n}}{h} .
\end{array}\right.
$$

A good choice for the pseudo-random sequence $\omega_{n}$ is the $\left(k_{1}, k_{2}\right)$ van der Corput sequence. In practice, we consider the $(5,3)$ van der Corput sequence.

The resulting scheme has the following properties [16]:

- it is stable in the sense of (17);

- it is conservative in a statistical sense;

- it is entropy dissipative in a statistical sense;

- it does not produce spurious oscillations at the two-fluid interface.

\section{Numerical results}

We can extend the scheme to higher dimensions with dimensional splitting (for more details we refer to [13]). It is remarkable that the same random number can be used for one time step in the $x$ and $y$ directions. It is also remarkable that despite dimensional splitting, the two-dimensional scheme converges towards the right solution without oscillation. Indeed, since the work of Colella [6], it was generally admitted that applying the dimensional splitting procedure to the Glimm's scheme leads to poor numerical results.

We present in Figure 1 the results of a two-dimensional shock-droplet simulation. The initial droplet is a disk. A shock-wave coming from the right of the computational domain interacts with the droplet. The computations have been realized thanks to an OpenCL/MPI implementation of the two-dimensional RIS scheme. For this test case, we use a cluster of four AMD Radeon HD 7970 GPU. The detailed description of the test case is given in [13]. We display the droplet after the interaction. We observe that we are able to capture a sharp interface. The numerical noise 
is moderate, despite the random nature of the scheme. Because we use a very fine mesh with $20,000 \times 5,000$ cells, we are able to zoom on small Kelvin-Helmholtz vortices (see Figures 2 and 3).

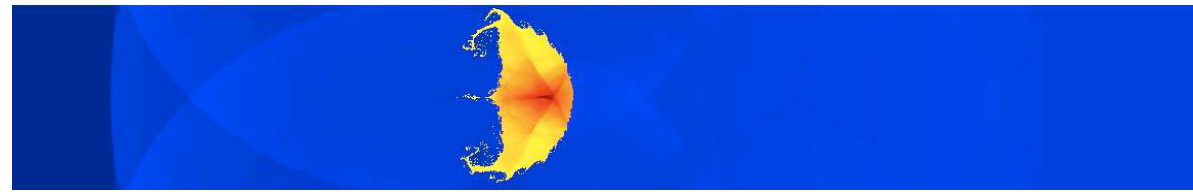

\section{$\mathrm{RHO}$}

1.22

4.41

7.6

Fig. 1 Shock-droplet simulation. Density plot. Full view of the computational domain.

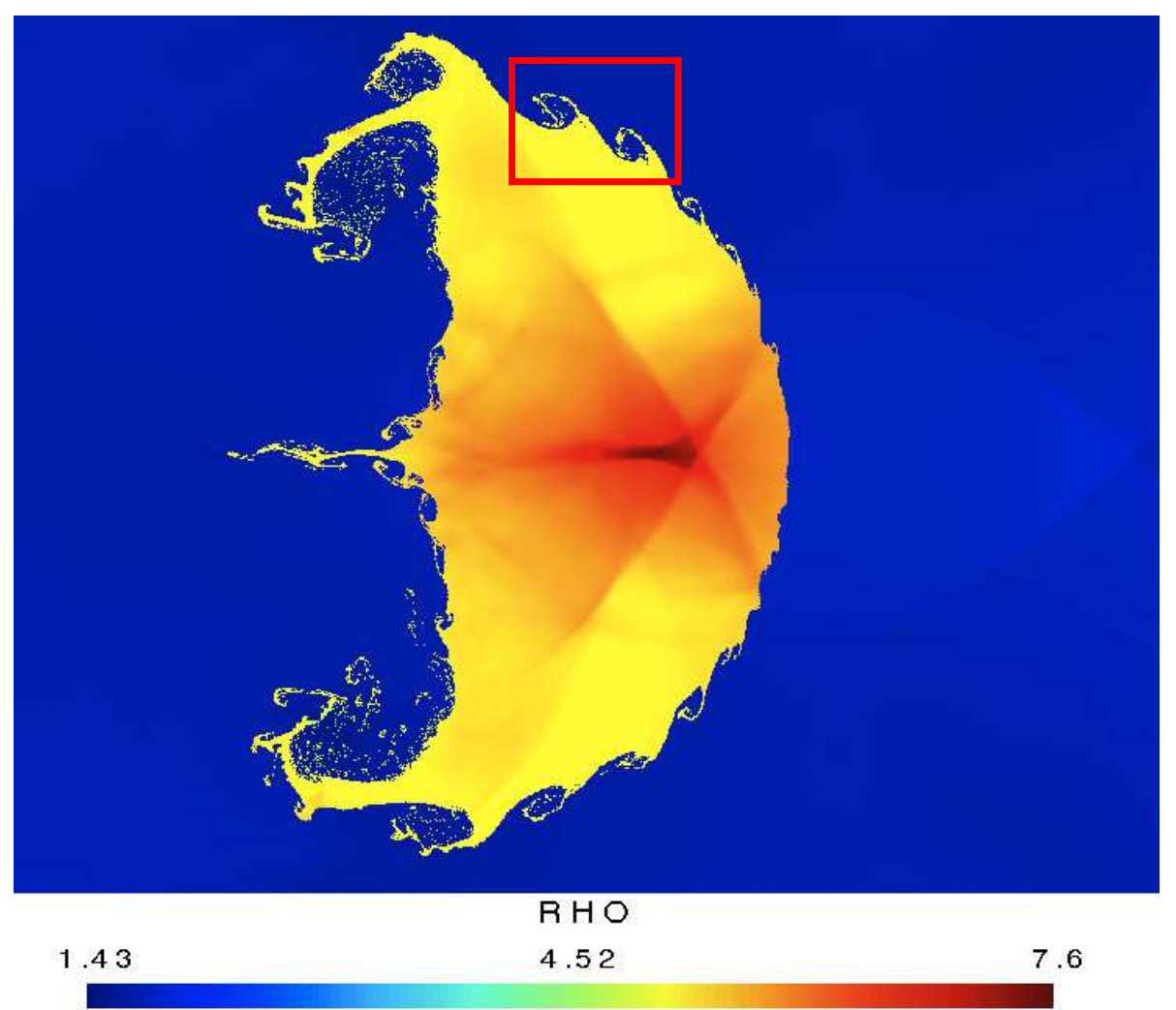

Fig. 2 Shock-droplet simulation. Density plot. Zoom on the droplet. 


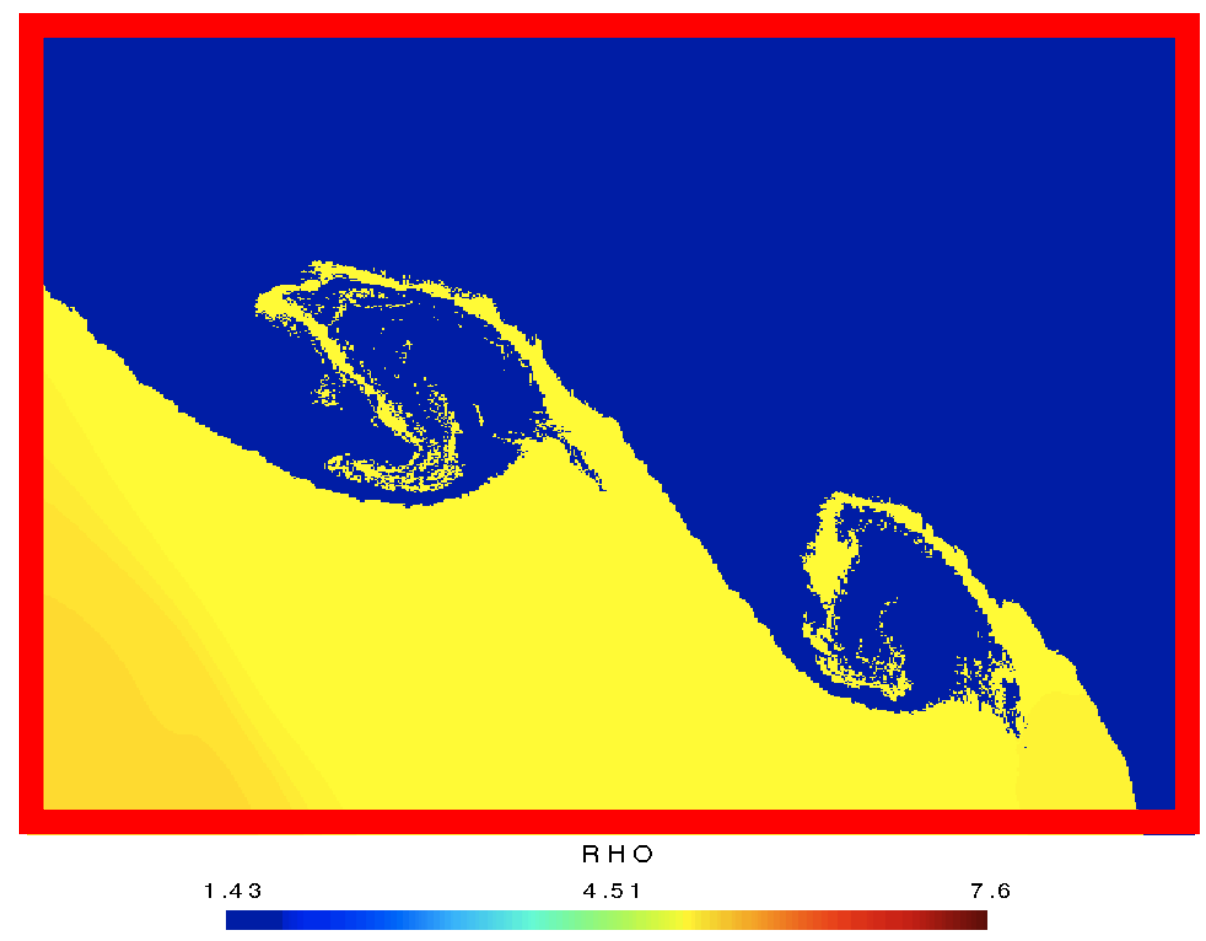

Fig. 3 Shock-droplet simulation. Density plot. Second zoom on the droplet.

We present in Figure 4 the results of a two-dimensional shock-bubble simulation. The initial bubble is a disk. A shock wave coming from the left of the computational domain interacts with the bubble. We display the bubble after that it has been split by the shock wave. The computations have been realized thanks to an OpenCL/MPI implementation of the two-dimensional RIS scheme. For this test case, we use a cluster of ten NVIDIA K20 GPU. The detailed description of the test case is given in [13]. We use a very fine mesh with $40,000 \times 20,000$ cells. As in the previous test case, we can zoom in order to observe small details of the split region (see Figures 5 and 6).

\section{Conclusion}

We have shown that a widely used two-fluid liquid-gas model has a non-convex hyperbolicity domain. This non-convexity can lead to the failure of the Godunov scheme after only one time step. This is true even if the continuous model admits a perfectly well defined solution that satisfies the Lax characteristic criterion. 


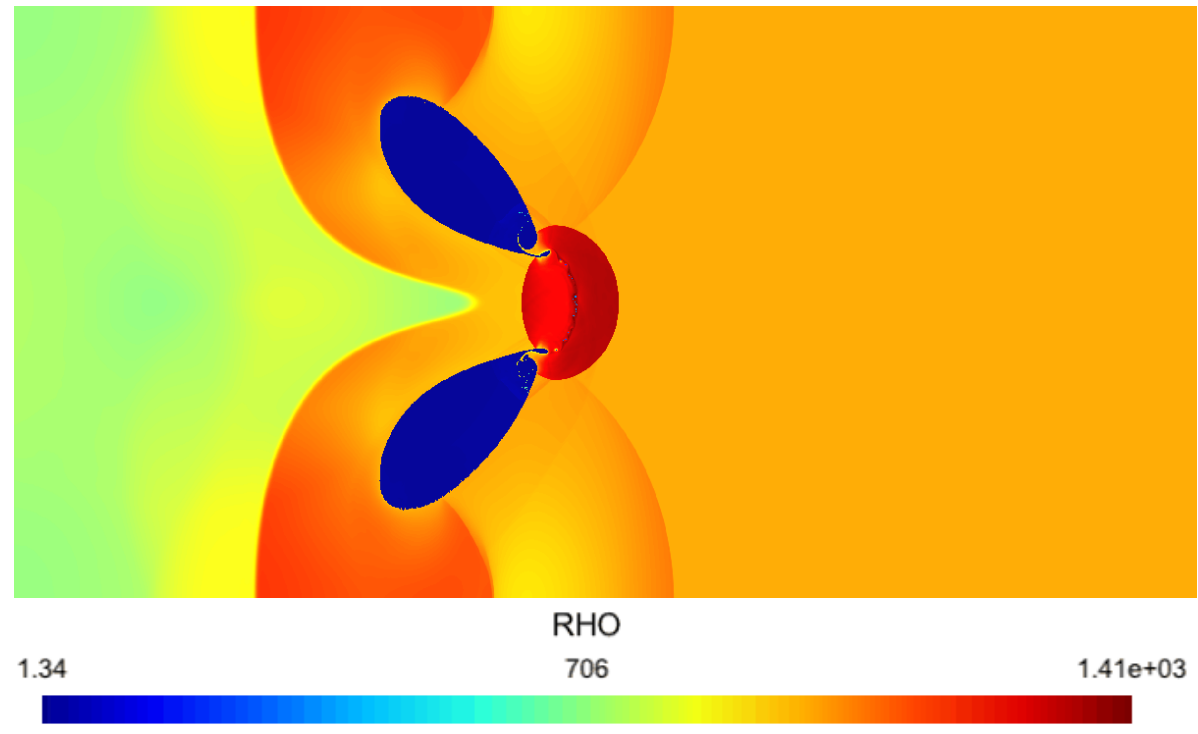

Fig. 4 Shock-bubble simulation. Density plot.

We have proposed a modified equation of state for recovering a convex hyperbolicity domain. The resulting pressure law is more complicated and is not a stiffened gas equation of state anymore in the pure liquid, when the gas mass fraction $\varphi=0$.

For keeping the simplicity of the stiffened gas equation, we have thus constructed a finite volume scheme, the RIS scheme, which avoids the numerical diffusion of the mass fraction. The RIS scheme is based on a Lagrangian finite volume approach coupled with a random sampling at the interface.

Let us conclude that the construction of a conservative finite volume scheme that would give accurate results at contact waves for one-fluid or two-fluid general pressure laws is still an open question.

\section{References}

1. T. Barberon, P. Helluy. Finite volume simulation of cavitating flows. Computers \& Fluids, 34 , $832-858$ (2005)

2. T. Barberon, P. Helluy, S. Rouy. Practical computation of axisymmetrical multifluid flows. Int. J. Finite Vol. 1(1), 1-34 (2004)

3. C. Chalons, F. Coquel. Capturing infinitely sharp discrete shock profiles with the Godunov scheme. Hyperbolic problems: theory, numerics, applications, 363-370, Springer, Berlin, (2008)

4. C. Chalons, F. Coquel. Computing material fronts with a Lagrange-Projection approach. http://arxiv.org/abs/1012.4561 (2010)

5. C. Chalons, P. Goatin. Transport-equilibrium schemes for computing contact discontinuities in traffic flow modeling. Commun. Math. Sci., 5(3), 533-551 (2007) 


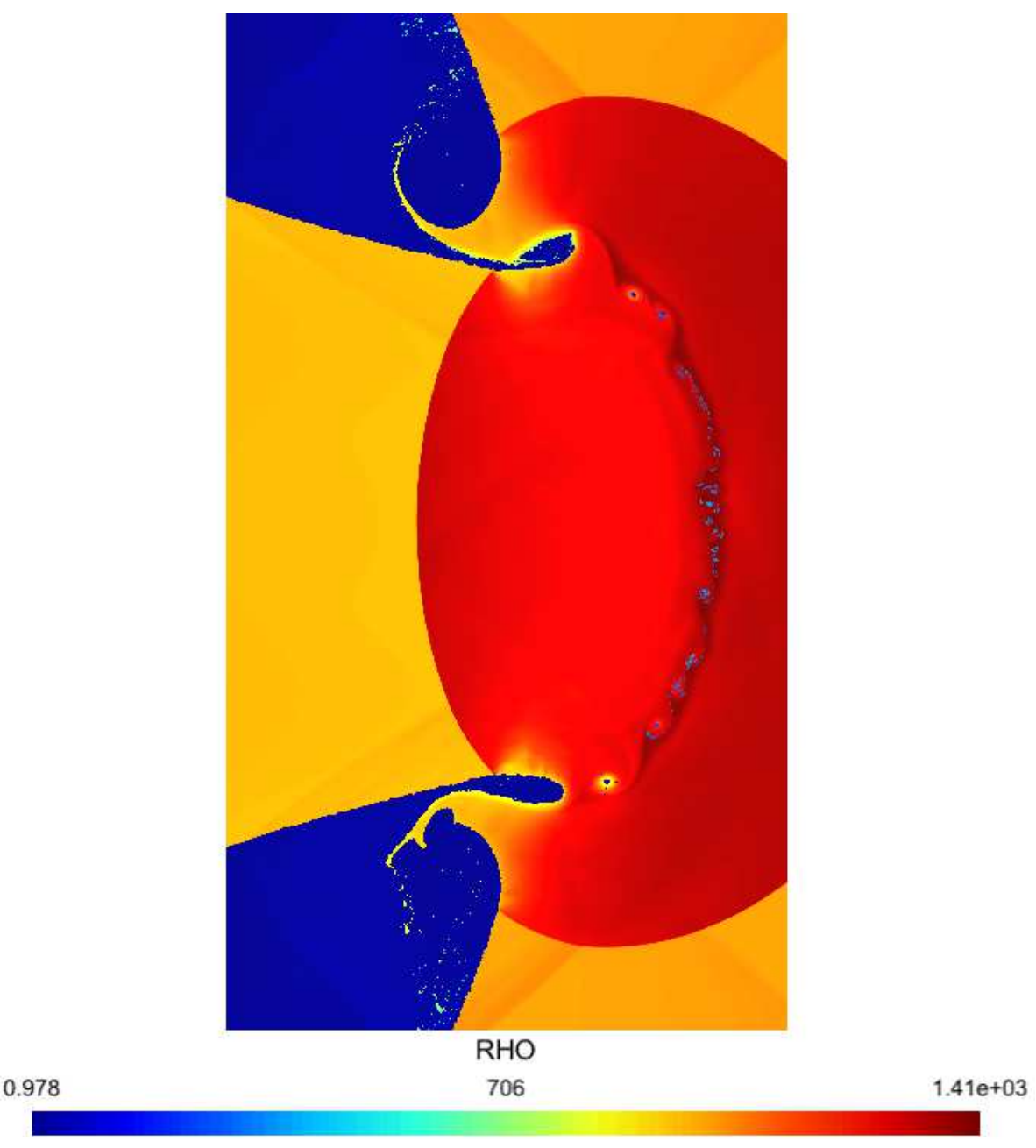

Fig. 5 Shock-bubble simulation. First zoom. Density plot.

6. P. Colella. Glimm's Method For Gas Dynamics. SIAM, J. Sci. Stat. Comput., 3(1) (1982)

7. J.-P. Croisille. Contribution à l'étude théorique et à l'approximation par éléments finis du système hyperbolique de la dynamique des gaz multidimensionnelle et multiespèces. $\mathrm{PhD}$ thesis, Université Paris VI (1990)

8. T. Gallouët, J.-M. Hérard, N. Seguin. A hybrid scheme to compute contact discontinuities in one-dimensional Euler systems. M2AN Math. Model. Numer. Anal. 36(6), 1133-1159 (2003).

9. J. Glimm. Solutions in the large for nonlinear hyperbolic systems of equations. Comm. Pure Appl. Math., 18, 697-715 (1965)

10. E. Godlewski, P.-A. Raviart. Numerical approximation of hyperbolic systems of conservation laws. Applied Mathematical Sciences, 118, Springer-Verlag, New York (1996)

11. F. Golay, P. Helluy. Numerical schemes for low Mach wave breaking. Int. J. Comput. Fluid Dyn. 21(2), 69-86 (2007) 


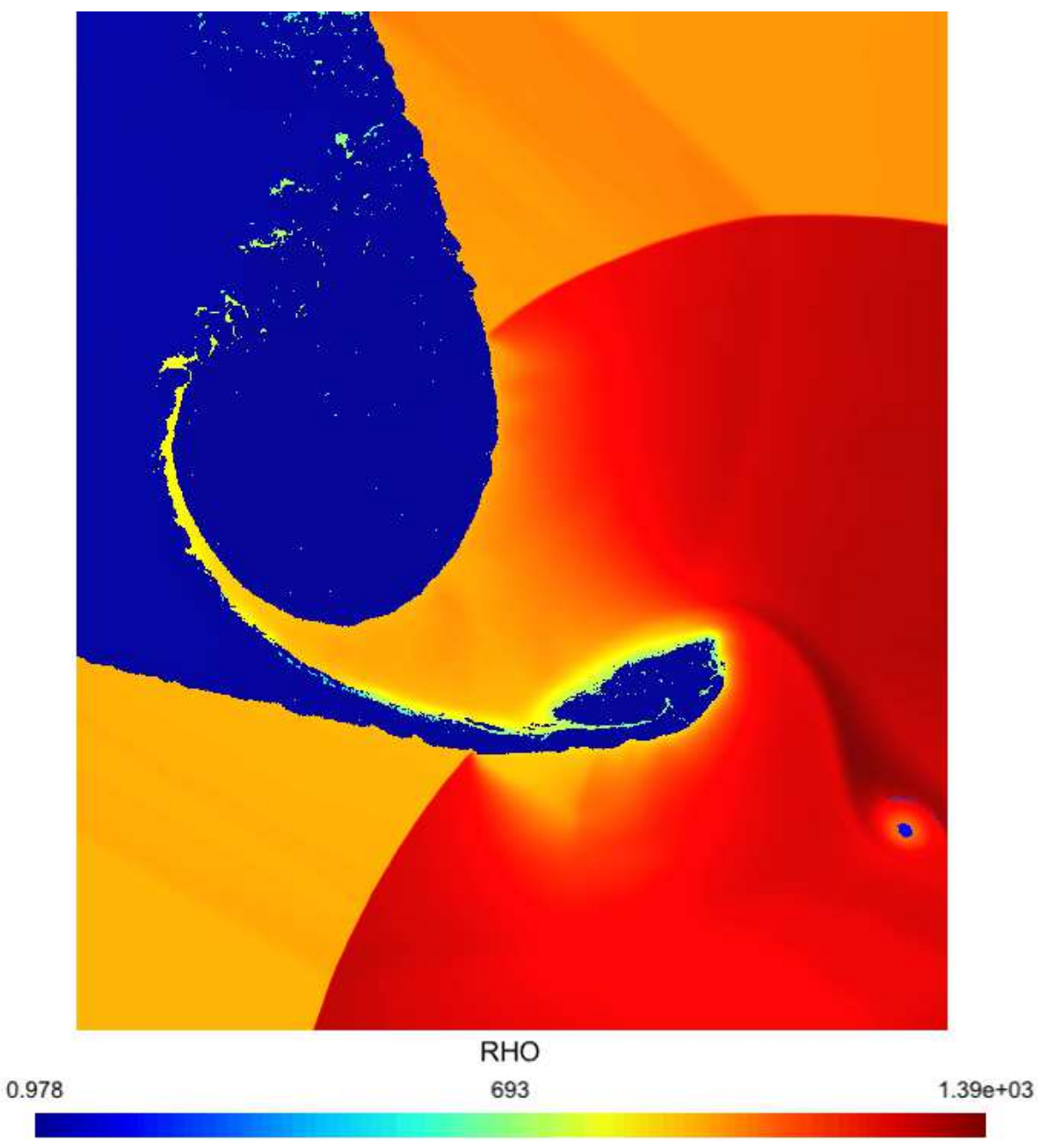

Fig. 6 Shock-bubble simulation. Second zoom. Density plot.

12. A. Harten, P. D. Lax, C. D. Levermore, W. J. Morokoff. Convex entropies and hyperbolicity for general Euler equations. SIAM J. Numer. Anal. 35(6), 2117-2127 (1998)

13. P. Helluy, J. Jung. OpenCL simulations of two-fluid compressible flows with a random choice method. Int. J. Finite Volumes, 10, 1-38 (2013)

14. P. Helluy, H. Mathis. Pressure laws and fast Legendre transform. Math. Models Methods Appl. Sci. 21(4), 745-775 (2011)

15. P. Helluy, N. Seguin. Relaxation models of phase transition flows. M2AN Math. Model. Numer. Anal. 40(2), 331-352 (2006)

16. J. Jung. Schémas numériques adaptés aux accélérateurs multicoeurs pour les écoulements bifluides. PhD thesis. University of Strasbourg (2013)

17. S. Karni. Multicomponent flow calculations by a consistent primitive algorithm. Journal of Computational Physics, 47, 1115-1145 (1994) 
18. P. D. Lax. Hyperbolic systems of conservation laws. II. Comm. Pure Appl. Math. 10, 537-566 (1957)

19. M. S. Mock. Systems of conservation laws of mixed type. J. Diff. Eq., 7, 70-88 (1980)

20. W. Mulder, S. Osher, and J. A. Sethian. Computing interface motion in compressible gas dynamics. J. Comput. Phys., 100(2),209-228 (1992)

21. S. Müller, P. Helluy, J. Ballmann. Numerical simulation of a single bubble by compressible two-phase fluids. Internat. J. Numer. Methods Fluids 62(6), 591-631 (2010)

22. R. Saurel and R. Abgrall. A simple method for compressible multifluid flows. SIAM J. Sci. Comput., 21(3), 1115-1145, (1999) 